\title{
Urogenital Symptoms and Pain History as Precursors of Vulvodynia: A Longitudinal Study
}

\author{
Barbara D. Reed, M.D., MSPH, Carolyn M. Payne, B.S., ${ }^{2 \star}$ Sioban D. Harlow, Ph.D., ${ }^{3}$ \\ Laurie J. Legocki, Ph.D., ${ }^{1}$ Hope K. Haefner, M.D., ${ }^{4}$ and Ananda Sen, Ph.D. ${ }^{1,5}$
}

\begin{abstract}
Background: We sought to assess vulvodynia incidence and risk factors among those with and without premorbid urogenital symptoms.

Methods: Women's Health Registry members who completed a baseline assessment in 2004 were sent a 2-year and 4-year follow-up survey containing a validated screen for vulvodynia. Subgroup analysis of vulvodynia incidence rates was performed, and risk factors associated with incidence were assessed.

Results: Of 1037 original enrollees, 723 (69.7\%) completed consecutive surveys (initial and 2-year or initial, 2year, and 4-year), 660 of whom did not have current or past vulvodynia at baseline. Of these $660,71(10.8 \%)$ first met criteria for vulvodynia within the 4-year period, for an annual incidence rate of $3.1 \%$ (95\% confidence interval [CI] 2.5-4.0). Baseline strict controls were less likely to develop criteria for vulvodynia diagnosis (annual incidence rate of $1.4 \%$ ) compared to those with an intermediate phenotype (presence of dyspareunia or history of short-term vulvar pain), for whom the incidence rate was 5.6\% $(p<0.001)$. Risk factors for incident vulvodynia differed between these two groups. Among the strict controls, an increased risk was noted among younger women (incidence rate ratio) [IRR] 3.6). For those with an intermediate phenotype, risk was increased among nonwhite women and those reporting pain with or after intercourse (IRR 2.2, 3.4, and 3.1, respectively). In both control groups, incident vulvodynia risk increased among those reporting urinary burning at enrollment (IRR 4.2 and 2.8 for strict and intermediate phenotype controls, respectively).

Conclusions: The annual incidence of vulvodynia is substantial (3.1\%) and is greater among women reporting a history of dyspareunia or vulvar pain that did not meet criteria for vulvodynia compared to those without this history, suggesting that generalized urogenital sensitivity may be a common underlying mechanism predating the clinical presentation of vulvodynia.
\end{abstract}

\section{Introduction}

V ULVODYNIA IS A DISORDER characterized by hypersensitivity at the vulva or vaginal introitus (vestibule) that typically causes sexual intercourse to be painful or intolerable. ${ }^{1}$ Its prevalence has been reported to be between $3.1 \%$ and $15 \%{ }^{2-5}$ Limited data are available on the incidence of vulvodynia in the general population ${ }^{6,7}$ and on the relationship between prior experiences with vulvar symptoms and onset of vulvodynia.

Cross-sectional studies have suggested that vulvodynia is associated with other pain symptoms and with comorbid medical conditions, such as fibromyalgia, chronic fatigue syndrome, recurrent vulvovaginal infections, and yeast in- fections. ${ }^{1,6,8-11}$ However, the cross-sectional study design does not allow differentiation between those symptoms that are precursors to vs. comorbid with vulvodynia. Few prospective data are available. ${ }^{6,7}$ A prior analysis from this current prospective study after 2 years of follow-up estimated the annual incidence of vulvodynia to be $1.8 \%$ among strict controls (defined as those without dyspareunia or a history of vulvar pain) ${ }^{6}$ and found younger age and a history of pain after intercourse to be associated with the new onset of vulvodynia. ${ }^{6}$ After 4 years of follow-up, the current analysis reassessed vulvodynia incidence rates among women who did not meet criteria for current or past vulvodynia at enrollment but who might not have been totally asymptomatic (a definition of greater relevance to the general population) and

\footnotetext{
${ }^{1}$ Department of Family Medicine, ${ }^{2}$ Undergraduate student, ${ }^{3}$ School of Public Health, ${ }^{4}$ Department of Obstetrics and Gynecology, and ${ }^{5}$ Department of Biostatistics, University of Michigan, Ann Arbor, Michigan.

*Present address: University of Toledo College of Medicine, Toledo, Ohio.
} 
evaluated the relationship between the presence of premorbid urogenital symptoms and the onset of vulvodynia during the study period.

Institutional review board approval for this study was obtained from the University of Michigan Medical Human Subjects Committee.

\section{Materials and Methods}

In 2004, 1037 members of the University of Michigan's Women's Health Registry completed either an online or a written survey regarding the presence of symptoms suggestive of vulvodynia. ${ }^{12}$ Further clinical investigation of a subset of those women demonstrated excellent reliability and validity of survey responses for predicting vulvodynia, ${ }^{12}$ and no further validation was performed in this study. In 2006 and 2008, follow-up surveys were sent to all women who had participated in the baseline assessment. Women with e-mail addresses were sent links to an online survey (SurveyMonkey.com, Portland, OR), and a written survey was mailed to all women who had no valid e-mail address and to those from whom no completed online surveys were received. A total of $723(69.7 \%)$ women completed the 2-year survey, ${ }^{6} 660$ $(63.6 \%)$ completed the 4-year follow-up survey, and 573 $(55.3 \%)$ completed all three surveys.

Participants were assigned to one of four clinical statuses, based on previously validated survey responses. ${ }^{12}$ Cases were defined as women having current vulvar pain (at the vaginal opening) that occurred over $\geq 3$ months. Past cases were defined as women who reported previous symptoms of vulvodynia but did not have them at present. Strict control status was assigned based on reporting no current pain with intercourse and no history of vulvar pain lasting $\geq 3$ months. The intermediate phenotype control category was composed of two subgroups: those women who reported pain with intercourse within the past 6 months but no vulvar pain and those who reported having had vulvar pain at some point but did not meet criteria for current or past vulvodynia (typically denying pain occurred for $\geq 3$ months). Those with data at consecutive rounds (initial and 2-year but no 4-year follow-up or initial, 2-year, and 4-year follow-up rounds) were included in the analysis $(n=723$, or $69.7 \%$ of the initial cohort). Of these, 660 (91.3\%) did not screen positive for vulvodynia at the initial round and could be evaluated for new onset vulvodynia over the course of the study.

Descriptive statistics were calculated for all variables. Characteristics (at enrollment) of the 660 women who completed consecutive surveys, compared to those who also did not screen positive for vulvodynia at the baseline but who did not complete consecutive surveys $(n=267)$, were assessed using chi-square analysis. The annual incidence rate and risk factors for vulvodynia overall and for various diagnostic subgroups were estimated using a negative binomial regression model (generalized linear model), with demographic and premorbid conditions included as independent variables, the logarithm of time (minimum of incidence-free observation time or time until incidence) used as the offset, and the annual incidence rate as the outcome.

\section{Results}

Characteristics of the potential participants for this analysis, stratified by whether they completed consecutive surveys, were compared (Table 1). This consisted of the 660 women
Table 1. Characteristics of Those Who Were Not a Case or Past Case at Enrollment $(N=927)$ and Who Did (Included in Subsequent Analyses) or Did Not (Excluded from Subsequent Analyses) Complete Consecutive Surveys

\begin{tabular}{|c|c|c|c|}
\hline Variable $^{\mathrm{a}}$ & $\begin{array}{c}\text { Consecutive } \\
\text { surveys } \\
(\mathrm{n}=660) \%\end{array}$ & $\begin{array}{c}\text { No consecutive } \\
\text { surveys } \\
(\mathrm{n}=267) \%\end{array}$ & value $^{\mathrm{p}}$ \\
\hline Age $\geq 50$ years & 42.6 & 33.2 & 0.009 \\
\hline Married & 59.8 & 54.4 & 0.130 \\
\hline White & 88.0 & 80.7 & 0.004 \\
\hline $\begin{array}{l}\text { Education level } \geq 16 \\
\text { years }\end{array}$ & 70.1 & 58.5 & 0.001 \\
\hline $\begin{array}{l}\text { Household } \\
\quad \text { income } \geq \$ 60,000\end{array}$ & 54.2 & 41.2 & $<0.001$ \\
\hline $\begin{array}{l}\text { Pain with first } \\
\text { intercourse }\end{array}$ & 26.1 & 20.2 & 0.064 \\
\hline Pain with first tampon & 30.5 & 36.8 & 0.081 \\
\hline \multicolumn{4}{|c|}{ Case-control status at enrollment } \\
\hline Controls & 56.4 & 63.7 & 0.041 \\
\hline $\begin{array}{l}\text { Intermediate } \\
\text { phenotype }\end{array}$ & 43.6 & 36.3 & \\
\hline
\end{tabular}

${ }^{a}$ Variables based on enrollment data.

${ }^{\mathrm{b}}$ Based on chi-square analysis.

included in the analysis who filled out consecutive surveys (including the initial and 2-year only [ $n=165]$ or the initial, 2 -year, and 4-year surveys $[n=495])$ and those who did not $(n=267)$ (Table 1). Compared to those lost to follow-up, those with consecutive data were more likely to be older and nonwhite and to report higher education and household income. They were also slightly more likely to have an intermediate symptom phenotype, compared to being a strict control, than were those who did not complete consecutive surveys.

\section{Prevalence of vulvodynia}

The proportion of participants completing consecutive surveys who met criteria for vulvodynia at the 2-year and the 4-year follow-up were $9.4 \%$ and $10.8 \%$, respectively. However, the specific individuals constituting the case or noncase subgroups differed between rounds. For example, of the 573 women completing both the 2-year and the 4-year follow-up rounds, $25(44.6 \%)$ of the 56 cases at the 2 -year round were still cases at the 4-year follow-up, with the rest no longer reporting ongoing symptoms.

\section{Incidence of vulvodynia}

The incidence rates of vulvodynia, categorized by enrollment case-control classification, are shown in Table 2. Over the 4-year follow-up period, of 660 participants who did not report current or past vulvodynia at the initial survey (ranging in age from 19 to 83 years), a total of 71 (10.8\%) developed symptoms of vulvodynia, for an estimated annual incidence rate of $3.1 \%$ (95\% confidence interval [CI] 2.5, 4.0). The annual incidence rate of vulvodynia among those defined as strict controls at enrollment was $1.4 \%$, similar to that reported previously using a similar definition of "strict control." ${ }^{\prime 6} \mathrm{We}$ further evaluated the incidence rates over the 4-year period among each of the diagnostic groups, including those with 
Table 2. Annual Incidence Rates of Vulvodynia

Among Those with Noncase Status at EnRollment

\begin{tabular}{lccc}
\hline & $\mathrm{n}$ & Number & $\begin{array}{c}\text { Incidence rate } \\
(\text { per 100 women } \\
\text { per year) }(95 \% \text { CI) }\end{array}$ \\
\hline $\begin{array}{l}\text { All control women who were not a case or past } \\
\text { case at enrollment }\end{array}$ & 660 & 71 & $3.1(2.5-4.0)$ \\
$\begin{array}{l}\text { Control women who had no pain with } \\
\text { intercourse and no history of vulvar pain }\end{array}$ & 372 & 18 & $1.4(0.9-2.2)$ \\
$\begin{array}{l}\text { Control women with intermediate phenotypes } \\
\text { b }\end{array}$ & 288 & 53 & $5.6(4.1-7.5)$ \\
\hline
\end{tabular}

\footnotetext{
${ }^{a}$ Using a negative binomial with log link model.

${ }^{\mathrm{b}}$ Intermediate phenotypes include those reporting pain with intercourse but no vulvar pain or a history of vulvar pain not lasting $\geq 3$ months.

CI, confidence interval.
}

intermediate symptoms at enrollment who did not meet criteria for vulvodynia (Table 2). The annual incidence rate among those with an intermediate phenotype at enrollment was substantially higher (annual incidence of 5.6\%) than that for the strict control group (annual incidence of 1.4/100 women), as shown $(p<0.001)$.

\section{Risk factors for incident vulvodynia}

Table 3 compares the baseline characteristics of those who became incident vulvodynia cases by the time of the 4-year follow-up in contrast to those who remained noncases, for all controls, and also stratified by whether they were strict controls or had an intermediate phenotype at the time of the initial survey. Shown are the results for the multivariable analyses, controlling for age, ethnicity, and income. Among the women in general, new onset vulvodynia was associated with a number of premorbid characteristics at baseline (including younger age $[<50]$, history of a yeast infection in the previous 2 years, pain reported with first tampon use or first intercourse, a history of pain with intercourse or pain after intercourse), with vulvar or urinary burning, with having an intermediate phenotype (as compared to being a strict control), and with a trend noted with genital itching. Among strict controls at enrollment, new onset vulvodynia was less likely in older women ( $\geq 50$ years) and more likely in those reporting urinary burning at enrollment. Among those with an intermediate phenotype, incident vulvodynia cases were more likely to occur among nonwhite women and among those reporting pain after intercourse or urinary burning or both on their enrollment survey.

\section{Discussion}

The incidence of vulvodynia, as diagnosed using validated survey-based criteria, ${ }^{12}$ is substantial, and occurs over a wide range of ages (23-78 years). However, this incidence rate varies considerably among women who fall into different noncase subgroups. Those categorized as strict controls at enrollment (no dyspareunia or past vulvar pain lasting $>3$ months) were less likely to develop vulvodynia (incidence rate $1.4 \%$ ) compared to those reporting dyspareunia without ongoing vulvar pain or those with a history of vulvar pain not meeting criteria for current or past vulvodynia (incidence rate $5.6 \%$ ). Given this increased risk, healthcare providers may wish to educate women with these symptoms about the characteristics of vulvodynia, thereby increasing the possi- bility of earlier identification and treatment should the symptoms progress to those consistent with this disorder.

Data on whether oral contraceptive (OC) use is associated with the presence of vulvodynia are conflicting, with some reports suggesting an association ${ }^{13-15}$ and others, including this study, suggesting none. ${ }^{16,17}$ Few account for whether the OCs were started before or after the development of the vulvar symptoms. We found no association between current use of OCs as was reported at enrollment with new onset of vulvodynia over the 4-year follow-up period.

Risk factors identified included not only those previously suggested, such as pain after intercourse, ${ }^{6}$ but also a newly identified risk factor of urogenital symptoms, such as vulvar burning and urinary burning, reported before the onset of vulvar pain meeting criteria for vulvodynia. A history of yeast infections has long been reported to be associated with vulvodynia. ${ }^{8,18}$ History of yeast infections was associated with risk of new onset vulvodynia only among those in the strict control group but not when controlled for age, ethnicity, and income. Because the diagnosis of yeast infections is often made based on symptoms alone or on in-office microscopic assessment without culture, the validity of this reported history remains uncertain ${ }^{19}$ and needs laboratory confirmation in a longitudinal study.

A history of pain after intercourse and the reported presence of genital symptoms, such as burning with urination, were associated with subsequent onset of vulvodynia among both strict controls and those with an intermediate phenotype. The presence of these symptoms before the diagnosis of vulvodynia suggests that a premorbid state of neuronal hypersensitivity in the introital area may exist that only later meets criteria for clinical vulvodynia.

There are limitations to this study. Validation of case status in the office was conducted previously on a subset of participants at the time of the baseline survey, ${ }^{6}$ but no further inoffice validation was performed for the 2-year and 4-year follow-up rounds. Based on previous validation studies, the probability of misclassification among a small proportion of participants based on concurrent infection or dermatologic disorders is thought to be small. ${ }^{1,12}$ In addition, although the cohort of women assessed in this series of surveys was not selected from a clinic-based population or from those with genitourinary symptoms, participation in the Women's Health Registry, from which the women in this study were enrolled, is voluntary and may not represent the population at large. 


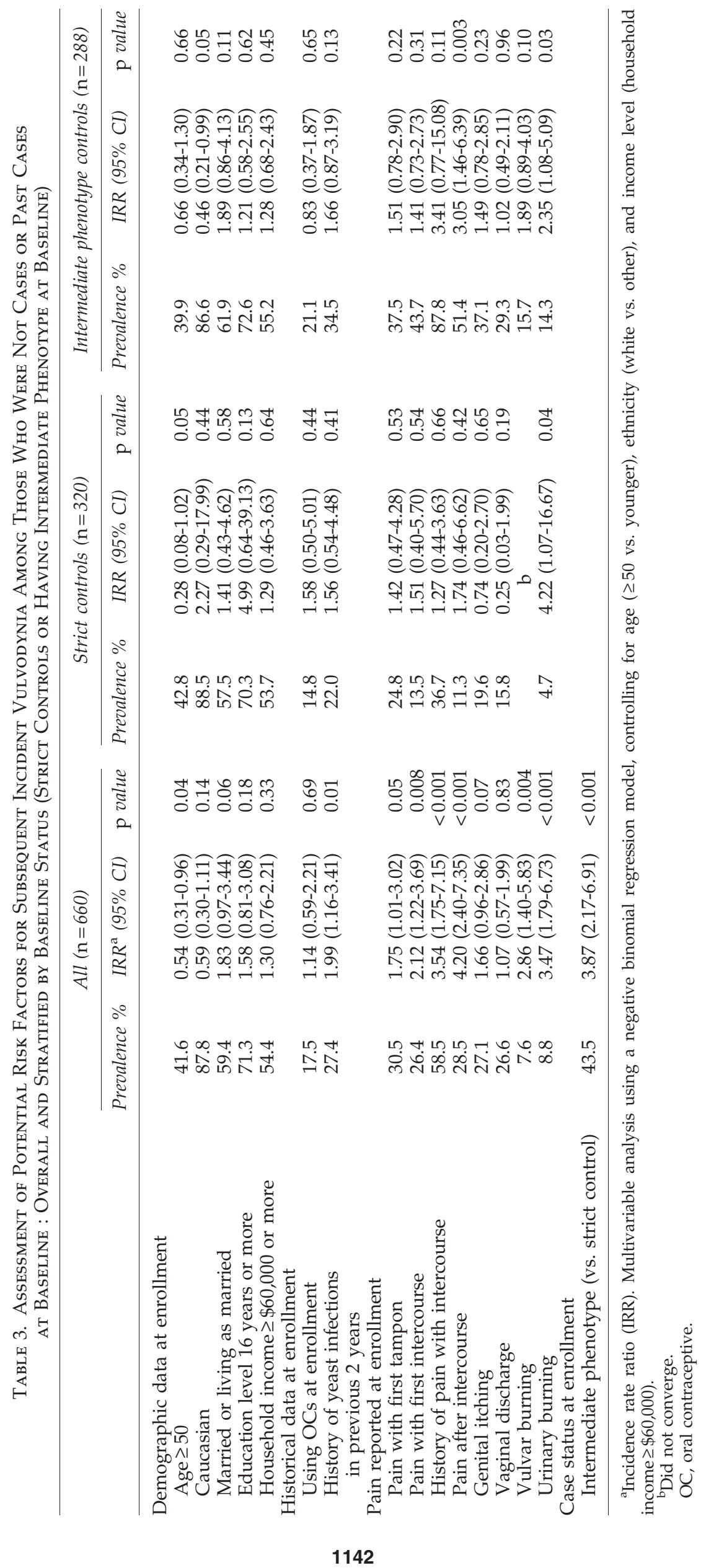




\section{Conclusions}

This 4-year follow-up study of women volunteers in a Women's Health Registry indicates that the annual incidence of vulvodynia over time is approximately 3\% and that a new onset of symptoms of vulvodynia is more likely to develop in women with intermediate symptoms of dyspareunia or with a history of short-term vulvar pain than in those denying this history. Further, women with new onset vulvodynia frequently have a premorbid history of nonspecific urogenital symptoms that may suggest neuronal hypersensitivity, which may allow detection of those at increased risk for subsequent vulvodynia.

\section{Acknowledgments}

We thank Sue Countryman for managing this project, Jill Bowdler for her survey management and secretarial assistance, and the Department of Family Medicine at the University of Michigan for partial funding of this project.

\section{Disclosure Statement}

No competing financial interests exist.

\section{References}

1. Harlow BL, Vazquez G, MacLehose RF, Erickson DJ, Oakes JM, Duval SJ. Self-reported vulvar pain characteristics and their association with clinically confirmed vestibulodynia. I Womens Health 2009;18:1333-1340.

2. Harlow BL, Wise LA, Stewart EG. Prevalence and predictors of chronic lower genital tract discomfort. Am J Obstet Gynecol 2001;185:545-550.

3. Reed BD, Crawford S, Couper M, Cave C, Haefner HK. Pain at the vulvar vestibule: A web-based survey. I Lower Genit Tract Dis 2004;8:48-57.

4. Bachmann G, Rosen R, Arnold L, et al. Chronic vulvar and other gynecologic pain: Prevalence and characteristics in a self-reported survey. J Reprod Med 2006;51:3-9.

5. Reed BD, Harlow SD, Sen A, et al. Prevalence and demographic characteristics of vulvodynia in a population-based sample. Am J Obstet Gynecol 2012;206:170 e171-179.

6. Reed BD, Haefner HK, Sen A, Gorenflo DW. Vulvodynia incidence and remission rates among adult women: A 2-year follow-up study. Obstet Gynecol 2008;112:231-237.

7. Sutton JT, Bachmann GA, Arnold LD, Rhoads GG, Rosen RC. Assessment of vulvodynia symptoms in a sample of
U.S. women: A follow-up national incidence survey. J Womens Health 2008;17:1285-1292.

8. Arnold LD, Bachmann GA, Rosen R, Kelly S, Rhoads GG. Vulvodynia: Characteristics and associations with comorbidities and quality of life. Obstet Gynecol 2006;107: 617-624.

9. Arnold LD, Bachmann GA, Rosen R, Rhoads GG. Assessment of vulvodynia symptoms in a sample of U.S. women: A prevalence survey with a nested case-control study. Am J Obstet Gynecol 2007;196:128 e121-126.

10. Zolnoun DA, Rohl J, Moore CG, Perinetti-Liebert C, Lamvu GM, Maixner W. Overlap between orofacial pain and vulvar vestibulitis syndrome. Clin J Pain 2008;24:187-191.

11. Reed BD, Harlow SD, Legocki LJ, Edwards RM, Sen A, Haefner HK. Vulvodynia and other chronic pain comorbidities. J Low Genit Tract Dis 2011;15:S14.

12. Reed BD, Haefner HK, Harlow SD, Gorenflo DW, Sen A. Reliability and validity of self-reported symptoms for predicting vulvodynia. Obstet Gynecol 2006;108:906-913.

13. Bazin S, Bouchard C, Brisson J, Morin C, Meisels A, Fortier M. Vulvar vestibulitis syndrome: An exploratory casecontrol study. Obstet Gynecol 1994;83:47-50.

14. Sjoberg I, Nylander Lundqvist EN. Vulvar vestibulitis in the north of Sweden. An epidemiologic case-control study. J Reprod Med 1997;42:166-168.

15. Bouchard C, Brisson J, Fortier M, Morin C, Blanchette C. Use of oral contraceptive pills and vulvar vestibulitis: A casecontrol study. Am J Epidemiol 2002;156:254-261.

16. Harlow BL, Vitonis AF, Stewart EG. Influence of oral contraceptive use on the risk of adult-onset vulvodynia. $\mathrm{IRe}-$ prod Med 2008;53:102-110.

17. Foster DC. Case-control study of vulvar vestibulitis syndrome. J Womens Health 1995;6:677-680.

18. Edwards L. New concepts in vulvodynia. Am J Obstet Gynecol 2003;189:S24-30.

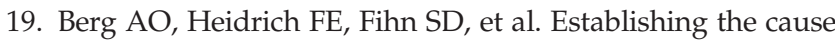
of genitourinary symptoms in women in a family practice. Comparison of clinical examination and comprehensive microbiology. JAMA 1984;251:620-625.

Address correspondence to:

Barbara D. Reed, M.D., MSPH University of Michigan

Department of Family Medicine 1018 Fuller Street

Ann Arbor, MI 48104-1213

E-mail: barbr@umich.edu 